\title{
INCINERATE, RECYCLE, OR WASH AND REUSE?
}

\author{
Martin A. Hubbe \\ What is the best way to minimize the environmental impact of using a \\ product such as paper? Three debating teams were formed within a \\ university class. One team advocated increased recycling of paper. \\ Another team pointed to evidence showing reduced environmental \\ impact and lower net $\mathrm{CO}_{2}$ emissions if the paper is incinerated rather \\ than recycled. A third team advocated the replacement of paper by \\ items such as porcelain plates and video screens, cutting costs and \\ reducing waste by multiple reuse.
}

Keywords: Paper recycling, Incineration, Reuse, Life cycle analysis, Pollution prevention, Environmental impact

Contact information: Department of Forest Biomaterial Science and Engineering, College of Natural Resources, North Carolina State University, Campus Box 8005, Raleigh, NC 27695-8005; hubbe@ncsu.edu

\section{ADVENTURES IN TEACHING ABOUT POLLUTION PREVENTION}

Values that are formed while one is very young often trump scientific arguments. Nevertheless, it is the job of faculty members, such as myself, to get university students to think about such issues as energy, toxic byproducts, and the value of relying on renewable resources.

In Spring 2003 I had the delightful opportunity to lead a session of "Advances in Pollution Prevention," a class offered to both on-campus and distance education students through NC State University's Engineering Online program. The class included about 22 undergraduate and graduate students, five of whom were participating by means of CDs, e-mail, and discussion forums. Working with Christine Grant, the course instructor, I had assigned students to one of three debating positions. Group 1 was asked to advocate the position that the proportion of paper that becomes recycled should be increased, in order to minimize environmental impacts. Group 2 was asked to advocate for increased incineration of used paper goods in order to minimize both energy use and the accumulation of landfill materials. Group 3 was asked to advocate for the replacement of disposable paper products by various forms of products designed for multiple reuse, sometimes involving washing of the product. In order to include the distance education students effectively in the debate, I assigned them the task of preparing written opening statements for each advocacy position.

The opening arguments introduced the main technical points supporting each of the three advocacy positions. Citing the EPA and various other sources, the team advocating "increased recycling" described how somebody's waste can be transformed into products, using up less landfill space, and reducing the consumption of wood. "You can pick the waste paper up at the curbside, just like trash," and "you avoid the toxic

Hubbe. (2007). "Incinerate, recycle, or reuse," BioResources 2(1), 1-2. 
emissions" associated with either primary pulp production or incineration of wastepaper. The opening statements in favor of "increased incineration of wastepaper" relied heavily upon life-cycle analyses, comparing de-inking to recycling. By incineration it was possible to avoid generation of toxic sludge, reduce overall air emissions, and displace the use of fossil fuels. The third team asserted that "replacing paper with reusable containers and surfaces" achieves superior environmental benefits and sharply reduces the needs for recycling, incineration, or other means of trash disposal. For instance, one porcelain cup can replace hundreds of paper cups.

After a few minutes of discussion within the groups, the on-campus students cautiously began to challenge and rebut statements made by opposing teams. The prorecycling team pointed out that the trees left in the forest continue to consume $\mathrm{CO}_{2}$, improving the air quality. Responding to the "reuse" group, they pointed out that reusable items are not necessarily reused, depending on people's habits. Washing of dishes increases the load on wastewater treatment facilities. The "reuse" group countered that high-volume washing facilities, as in a restaurant, can use detergent and hot water quite efficiently, and the chemicals required for de-inking of pulp are a greater environmental hazard. The pro-incineration team continued to cite results of studies indicating a lower net emission of toxic fumes and a lower overall cost compared to the other alternatives.

\section{TAKING STOCK}

Judging from the rising energy in the room, today's students care a lot about environmental issues. One student pointed out the somewhat artificial nature of the debate, suggesting that the best approach probably would involve a combination of the three debating positions. Dr. Grant added that different situations, e.g. a fast food restaurant $v s$. cooking or studying at home, might favor completely different strategies to minimize the environmental impact of paper use. It was interesting to note that only one of the on-campus students ultimately voted in favor of the incineration option, even though that team had offered the strongest evidence, based on life-cycle analysis. At least five students continued to favor paper recycling at the end of the class session. One of those students mentioned how strongly they had been influenced by presentations that they had heard while in Elementary School. But the largest number, over seven students, declared themselves to be in favor of "source reduction and reuse" at the end of the class.

Dr. Grant urged the students to hold onto their experience of looking carefully at the details and critical arguments for and against different environmental policies. "Some day soon you may find yourself in a position of influence, and you can help formulate policies in a more beneficial way." I urged the students to be especially careful when comparing different sources of energy; renewable energy can have environmental and societal benefits, compared to sources such as coal, petroleum, and nuclear energy. People vary too, with habits ranging from "lazy and cheap" to "crazy about the environment." In order to maintain progress in research areas that are of concern to readers of this magazine, it is essential that each of us take steps to tell our neighbors about both the benefits of and the need to protect our renewable resources.

Hubbe. (2007). "Incinerate, recycle, or reuse," BioResources 2(1), 1-2. 